\title{
Techno-Economic Evaluation of FTTH Migration for a Network Provider: Comparison of NG-AON and TWDM-PON
}

\author{
Marlies Van der Wee ${ }^{(1)}$, Koen Casier ${ }^{(1)}$, Kun Wang ${ }^{(2)}$, Sofie Verbrugge ${ }^{(1)}$, Mario Pickavet ${ }^{(1)}$ \\ (1) Ghent University - iMinds, Ghent, Belgium. Marlies.VanderWee@intec.ugent.be \\ (2) Acreo Swedish ICT, Kista, Sweden
}

Abstract This paper studies the business case of migration from legacy FTTH networks like active star Ethernet and GPON towards NG-AON and TWDM-PON for a network provider, evaluating its techno-economic viability and suggesting possible improvements.

\section{Introduction and motivation}

It is clear that all-fiber networks are the future for telecommunications access. Although the current technologies, such as active star Ethernet or active optical network (AON) and gigabit passive optical network (GPON), are capable of offering significant speeds, ever increasing future demands will require higher, symmetrical bandwidths. Furthermore, the deployment of all-fiber networks requires high investments, mostly in the deployment of the passive infrastructure (up to $70 \%$ of deployment cost [1]), while the active equipment for lighting up the fiber - only - accounts for $30 \%$. When however looking at the lifetime of both layers, the passive infrastructure is predicted to have a lifetime of 30 to 50 years, comparable to the lifetime of the traditional copper-based networks. The active equipment technology has a much shorter replacement period, e.g. 10 years. It is therefore mandatory to investigate the full longterm technology evolution and replacement when comparing FTTH investments. This paper will investigate the business case for a network provider (NP), responsible for installing and maintaining the active equipment, for an upgrade of two architectures: Next generation AON (NG-AON), migration from AON, and time and wavelength division multiplexing (TWDM) PON, migration from GPON [2].

\section{NG-AON and TWDM-PON}

NG-AON (Fig. 1a) is the natural evolution of an AON, active star architecture in which the customer has already been connected by means of Ethernet switches (32 ports switches are used in the study) from the central office (CO) via an intermediate aggregation point at a remote node/cabinet. One of the NG-AON variations is to adopt WDM-PON technology which can be used to backhaul the existing AON active star architecture. In a migration towards a node consolidation strategy [3], the first level of (or legacy) COs in the current FTTH network will be closed down, and hence, the OLTs and other equipment in the $\mathrm{CO}$ must be relocated further to an aggregated central access node (CAN). In relation to the NG-AON solution, arrayed waveguide gratings (AWG, 1:40) are placed in the legacy $\mathrm{CO}$ locations to backhaul all the traffic and forward them to the CAN. In total, this NG-AON can serve up to $32 \times 40$ (1280) customers from one feeder fiber. The migration from the existing AON active star to NG-AON (WDM PON backhauling) architecture can be deployed smoothly without changing the fiber infrastructure in the existing distribution network. Some NP equipment in the existing AON architecture (e.g. optical network terminals (ONTs) and Ethernet switches) can be reused in the NG-AON.

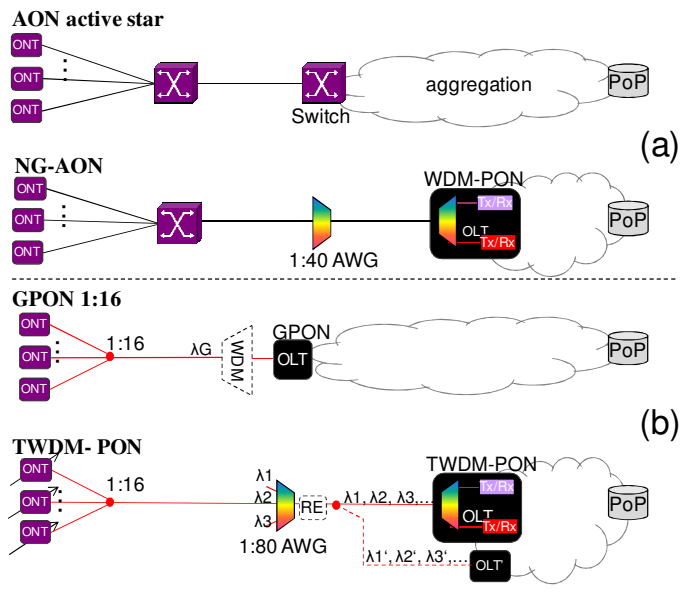

Fig. 1: Architecture changes for (a) migration from active star AON to NG-AON, and (b) migration from GPON 1:16 to TWDM-PON

TWDM-PON (Fig. 1b) is a natural evolution of a TDM-PON in which the customer is connected to the $\mathrm{CO}$ by means of a power splitter (1:16). As TWDM-PON solution, we assume that 80 of the existing 1:16 TDM-PONs are gathered further in the network by means of a 1:80 AWG. In this way each TDM-PON has its own wavelength and serves in turn 16 customers in a TDM manner. In total, a TWDM-PON80x16 will serve up to 1280 customers from one feeder fiber.

This paper will compare the migration costs of NG-AON and TWDM-PON, and indicate how 
they can be made competitive.

\section{Techno-Economic model for the NP}

The Techno-Economic model for the NP is based on an extension of the total cost of ownership (TCO) model of the TONIC tool [4]. The model includes both upfront and operational costs, and makes the distinction between general capital and operational expenditures (CapEx, OpEx), service provisioning and customer premises equipment (CPE installation and maintenance of the ONT). CapEx include the purchase and installation cost of the equipment in the $\mathrm{CO}$ and remote nodes, while OpEx refer to maintenance and energy consumption of this equipment. Patching and administrative costs related to a new subscription are grouped under service provisioning.

As mentioned before, we only focus on the NP cost in this paper. We exclude deployment, operations and possible upgrading of the passive infrastructure (e.g. trenches, fibers, ducts, splitters) from this investment analysis. We therefore make abstraction of these costs, as well as the revenues needed to achieve return on investment. We furthermore assume that the "old" GPON or AON equipment has been amortized.

\section{Results for the reference scenarios}

Within the European FP7-OASE project, nine reference scenarios were set up, based on the combination of a forecasted take-up rate (conservative, likely, aggressive) and a node consolidated area type (dense urban - DU, urban - $U$, rural - R). The reference areas are characterized by a number of households and a surface (Table 1). The adoption curves are based on a forecast for three representative European countries: the Netherlands (likely), Slovakia (aggressive) and Germany (conservative) [5].

Table 1: Parameters for the area types

\begin{tabular}{|l|l|l|}
\hline Area & Number of households & Surface $\left(\mathrm{km}^{2}\right)$ \\
\hline DU & 44,500 & 14 \\
\hline U & 51,000 & 142 \\
\hline R & 33,000 & 615 \\
\hline
\end{tabular}

Since we consider two technologies that result from a natural evolution of typical, current deployments (Active Ethernet AON and TDM$P O N$ ), we assume a migration towards the new technologies in 2020 (hard migration of all connected customers in one year), as this provides a fair basis for the cost comparison. We will therefore only study the business case from 2020-2030, and make abstraction of the costs incurred in previous periods.
The reference scenario is further complemented by a planning horizon of 10 years (the assumed lifetime of active equipment technology), a discount rate of $10 \%$, an energy cost of $0.21 €$ per $\mathrm{kWh}$ and a labor cost of $58 € /$ hour, all in accordance to the Belgian situation [6].

When calculating the TCO for both migrations, and taking into account a payback of investment within 10 years, it is possible to determine the needed average revenue per user (ARPU) for each reference scenario (Fig. 2).

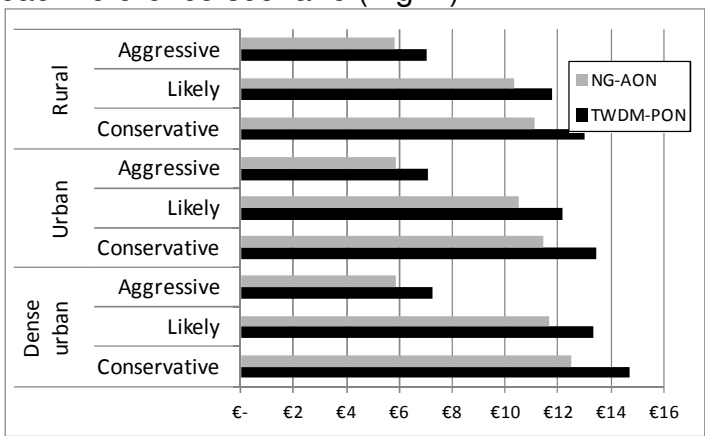

Fig. 2: Needed monthly ARPU to turn break-even after 10 years, for both migration steps

From this analysis, it is clear that the TCO for migrating from GPON to TWDM-PON is higher than the migration from AON to NG-AON. This can mainly be explained by the need for replacement of the GPON ONTs, while the original AON ONT can be reused. The AON ONT will also need replacement somewhere in the lifetime, but this exchange should not be performed at once.

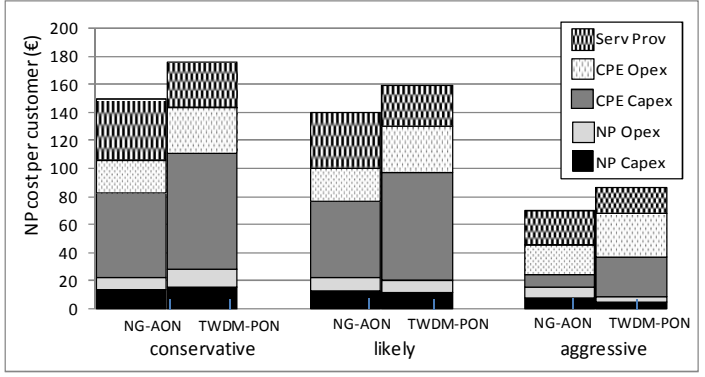

Fig. 3: Breakdown of the yearly cost per customer for a network provider (Dense Urban area)

The CPE CapEx and OpEx (replacement of ONTs for existing customers, new ONTs for new users and in-house installation, maintenance and energy consumption for both) therefore takes up a much higher share of total cost in TWDM-PON than in NG-AON (30-50\% versus $10-40 \%$ respectively, depending on the adoption curve).

The impact of the adoption curve is also interesting: the higher the initial adoption in time of migration, the lower the needed ARPU, which can be justified by the higher number of 
subscribers sharing part of the upfront investment (NP CapEx - purchase and installation of $\mathrm{CO}$ equipment, as well as CPE CapEx - sharing of in-building installations, see Fig. 3). Furthermore, the effect of statistical multiplexing comes into play for the NP CapEx, resulting in a higher cost per customer for $A O N$ for the aggressive curve. The results for the likely and conservative curve show less difference than likely and aggressive, but this can be easily explained by a smaller difference between those adoption curves [5].

Finally, we see a higher service provisioning cost per customer in NG-AON, which can be explained by the higher cost of physical provisioning required at remote nodes.

\section{How to improve the business case for TWDM-PON}

When comparing the ARPU found above with current FTTH offer prices, we can conclude that the business case for TWDM-PON migration will most probably not hold. It is therefore appropriate to search for improvements.

Since a large part of the TCO can be attributed to the necessary replacement of the ONTs in the PON migration case, recouping these costs in another way might help to reduce the monthly needed ARPU. One option is to charge an upfront fee, marketed as a necessary installation fee for upgrading the customer's subscription. Charging customers $€ 100$ installation fee (which corresponds in order of magnitude to the cost of a TWDM-PON ONT), reduces the needed monthly ARPU to €6 - 12 (in line with NG-AON). A second option to improve the business case is to spread the cost of migration over time (referred to as 'soft migration'). In this case, all new customers are connected directly to the new technology, while the existing customers are left with the choice for upgrading. In this way, the investment is spread out over a number of 'soft migration years', after which a forced migration upgrades the remaining customers on the old technology. In order to avoid parallel operations of two technology generations, the soft migration period should be kept limited in time. However, for the TWDMPON architecture under study here, the extra cost of this co-existence is limited (introduction of a migration specific WDM coupler per TDMPON during the maintenance window), so we study the impact of soft migration over the entire planning horizon (1-10 years) for the exemplary case of an urban area with aggressive adoption (Fig. 4). The impact of the soft migration is clearly shown as a shift (and reduction, due to the effect of discounting) of the cost migration peak to a later stage. This may lead to savings of $3 \%$ for a 5 -years-, and even $5 \%$ for a 10 years- soft migration period.

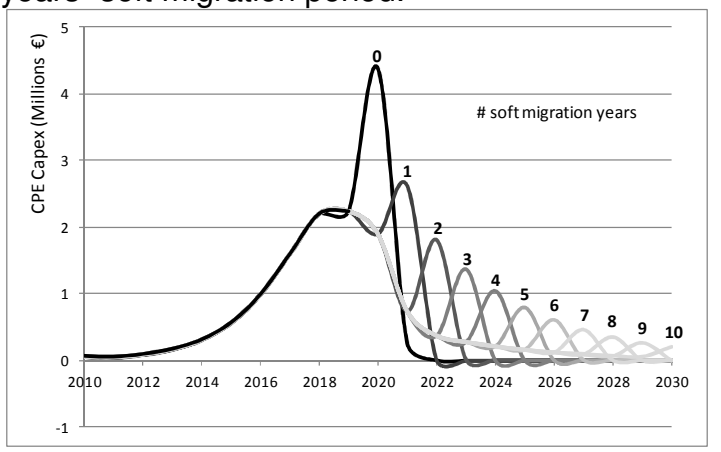

Fig. 4: Prolonging the soft migration period shifts and lowers the investment peak for the CPE Capex

\section{Conclusions}

This paper investigated the business case of a network provider that upgrades an existing AON or GPON FTTH infrastructure to a next generation infrastructure: NG-AON and TWDMPON respectively. Although the initial CapEx investment in AON infrastructure might be higher than the investment in PON, this paper clearly shows that upgrading AONs is cheaper than upgrading PONs. On the other hand, if the costs for CPE equipment (ONT, optical socket and installation) can be recouped in another way or spread out over time, this significantly ameliorates the business case for PON upgrade and makes it again competitive to AON.

\section{Acknowledgements}

This research was partially carried out in the framework of the project OASE (FP7/2007-2013) under grant agreement $n^{\circ} 249025$. The first author is funded by an IWT grant. The authors want to thank Mario Kind (DTAG) for reviewing the paper.

\section{References}

[1] Casier, K. et. al. (2008) A clear and balanced view on FTTH deployment costs. ITP journal, 2(3), 27-30.

[2] OASE (2012) Co-operation Models. Deliverable.

[3] Breuer, D. et al. (2010) Architectural options and challenges for next generation optical access. ECOC

[4] Mas, C. et al. (2012) Cost-based assessment of NGOA architectures and its impact in the business model. CTTE.

[5] Verbrugge, S. et al. (2012) Some insights in regulation and potential profitability of passive fiber infrastructure in Europe. ITS World.

[6] OECD Statistics, http://www.oecd.org/statistics/ 